\title{
Giant Angiomyolipoma Masquerading as Perinephric Abscess: A Diagnostic Conundrum
}

\author{
Sudhir Jain, Adiba Nizam*, Tariq Hameed, Astha Nayyar \\ Department of Surgery, Maulana Azad Medical College and Associated Lok Nayak Hospital, New Delhi, India \\ Email: sudhirkumar11@gmail.com, ${ }^{*}$ canadirocks@gmail.com
}

How to cite this paper: Jain, S., Nizam, A., Hameed, T. and Nayyar, A. (2021) Giant Angiomyolipoma Masquerading as Perinephric Abscess: A Diagnostic Conundrum. Open Journal of Urology, 11, 351-357. https://doi.org/10.4236/oju.2021.1110034

Received: January 27, 2021

Accepted: October 25, 2021

Published: October 28, 2021

Copyright $\odot 2021$ by author(s) and Scientific Research Publishing Inc. This work is licensed under the Creative Commons Attribution International License (CC BY 4.0).

http://creativecommons.org/licenses/by/4.0/

\begin{abstract}
Renal angiomyolipomas are the most common benign tumours of the kidney accounting for up to $1 \%$ of all renal masses. Giant angiomyolipomas which have a size greater than 10 centimetres are rare entities with few cases reported in literature. Small angiomyolipomas are usually a symptomatic and increasing size correlates with symptomatology. These are usually incidentally detected or when symptomatic may present with an abdominal lump, flank pain or hemorrhage. Herein, we report a rare case of 45 -year-old lady with giant angiomyolipoma with clinical presentation indistinguishable from perinephric abscess. The case is rare with regards to the large size of tumour and the discordant presentation unusual for an angiomyolipoma.
\end{abstract}

\section{Keywords}

Angiomyolipoma, Giant Angiomyolipoma, Perinephric Abscess

\section{Introduction}

Renal angiomyolipomas are the most common benign tumours of the kidney accounting for up to $1 \%$ of all renal masses. Giant angiomyolipomas which have a size greater than 10 centimetres are rare entities with few reported cases in literature. Small angiomyolipomas are usually asymptomatic and increasing size correlates with symptomatology. These are usually incidentally detected or when symptomatic may present with an abdominal lump, flank pain or hemorrhage. Here, we present a case that was misdiagnosed as perinephric abscess due to its peculiar presentation. The case is rare with regards to the large size of tumour and the discordant presentation unusual for angiomyolipoma.

\section{Clinical Presentation}

A 45-year-old lady presented to the emergency department with complaints of 
fever, left flank pain and burning micturition for duration of one week. She had been on thyroxine $50 \mathrm{mcg}$ for hypothyroidism for 8 years and was euthyroid. She had no other significant past medical or family history. Physical examination revealed pallor and a tender renal lump in left lumbar region. She had haemoglobin level of $8.4 \mathrm{~g} \%$, leukocyte count of $28,600 / \mathrm{mm}^{3}$, blood urea of $90 \mathrm{mg} \%$ and serum creatinine of $2.1 \mathrm{mg} \%$. Urine routine microscopy showed $8-10$ pus cells/hpf. Urine culture was sterile. Ultrasound of the abdomen showed a heterogeneously hypoechoic collection of dimensions $9.5 \times 5.6$ centimetres in left perinephric region visualised separately from the left kidney. An initial diagnosis of left perinephric abscess with acute kidney injury was made and management was instituted for the same. Patient was admitted for a course of IV antibiotics and therapeutic drainage of the abscess. Upon attempted diagnostic aspiration, only 5 millilitre of clotted blood was obtained. The initial diagnosis was called into question in this new light. Patient's kidney function improved with adequate hydration. A contrast enhanced computed tomography (CECT) scan was done for confirmation following normalisation of kidney function. CECT revealed giant left renal angiomyolipoma with large intra-tumoral bleed and an arterial feeder arising from left kidney (Figure 1).

Patient underwent selective arterial embolization which was unsuccessful. The patient was then planned for open exploration. Left simple nephrectomy was performed as nephron sparing nephrectomy was not deemed possible in this case due to large size of tumour encasing whole of the left kidney and intraoperative bleeding. The tumour measured $27 \times 8.5 \times 6$ centimetres and weighed 2000 grams. On gross examination of cut section of the tumour specimen, a large area of intra-tumoral haemorrhage was identified (Figure 2).

Histopathology reiterated angiomyolipoma of the left kidney as the definitive diagnosis. Postoperative period was uneventful and patient was discharged on postoperative day 5 (Figure 3). Patient followed up in outpatient department for 6 months and was found to be doing well. Patient gave her full and informed consent for publication of case report.

\section{Discussion}

Angiomyolipoma (AML) is a tumour consisting of thick-walled poorly organised blood vessels, smooth muscle $s$ and varying levels of mature adipose tissue [1]. Renal angiomyolipoma (AMLs) account for $1 \%$ of all renal masses [2] with overall incidence in general population of $0.07 \%-0.3 \%$ [3]. Giant angiomyolipoma which have a size greater than 10 centimetres [4] are rare entities with few reported cases in literature of AMLs measuring greater than 20 centimetres.

Up to $80 \%$ AMLs are sporadic. The classical sporadic presentation is of a middle-aged lady, suggesting a hormonal component to tumour growth, with a single asymptomatic tumour as was in this case [5]. AML may occur with tuberous sclerosis complex (TSC) in $20 \%-30 \%$ patients and approximately $50 \%$ of patients with TSC develop angiomyolipoma [6]. 

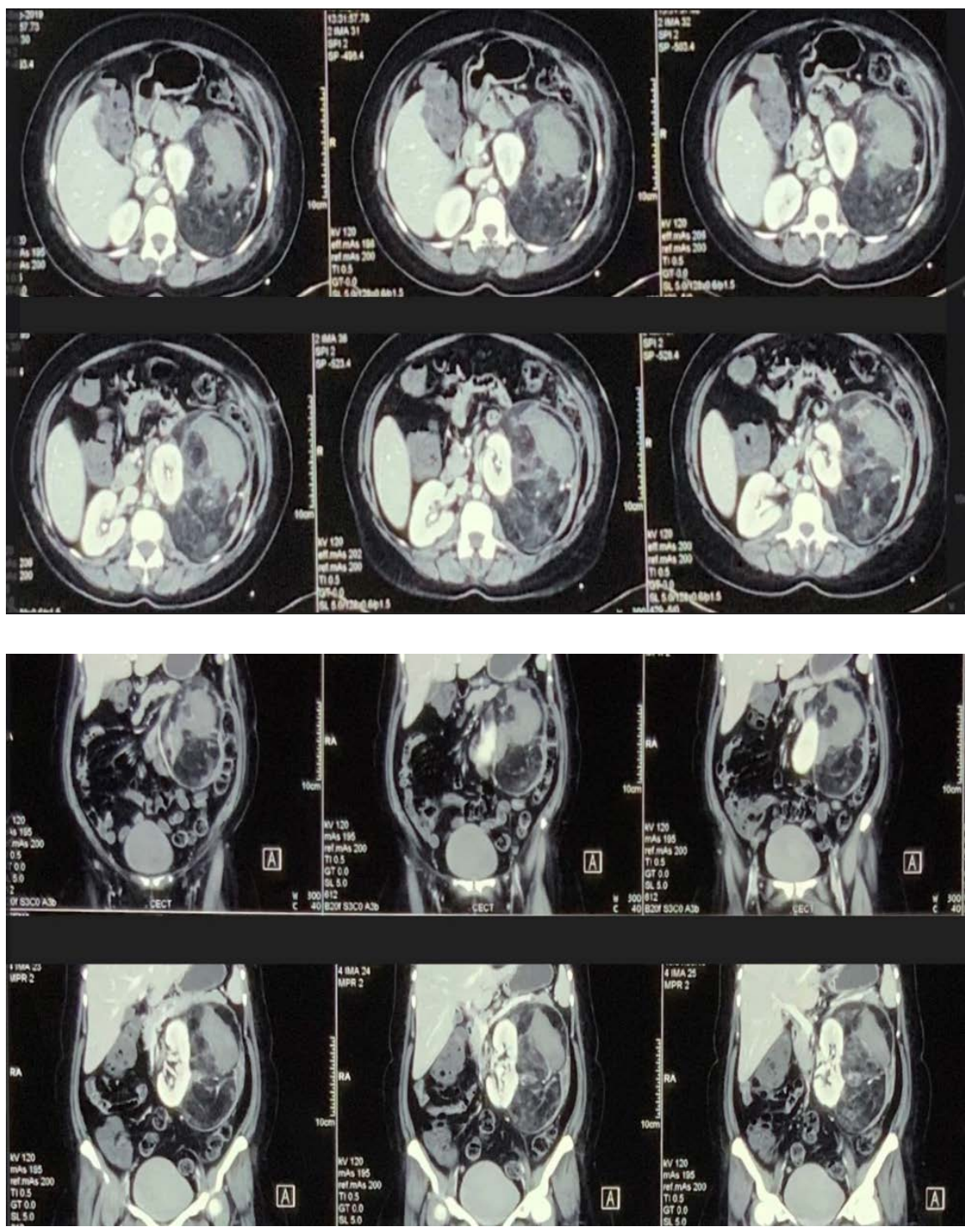

Figure 1. Axial and coronal views of contrast enhanced computed tomography showing large mass arising from left kidney laterally indenting its surface with negative 20 Hounsfield values (suggestive of fat) with a large area of hemorrhage within the tumour.

Asymptomatic tumours are usually less than 4 centimetres in size. Larger tumours tend towards symptomatic end of the spectrum which may include an abdominal mass, flank pain, haematuria, anaemia and haemorrhage. Life threatening intra-tumoral and retroperitoneal haemorrhage (Wunderlich syndrome) leading to shock may occur with large sized angiomyolipoma [7]. As seen in the presented case, wherein a giant angiomyolipoma presented with Wunderlich syndrome.

With the increased use of cross-sectional imaging over $80 \%$ of AMLs are now discovered incidentally, with haemorrhage at presentation (Wunderlich syndrome) seen in less than $15 \%$ and shock less than $10 \%$. The classic triad of symptoms associated with renal masses of flank pain, palpable mass and haematuria were historically found in $37 \%-41 \%$ of patients with AML [8]. 

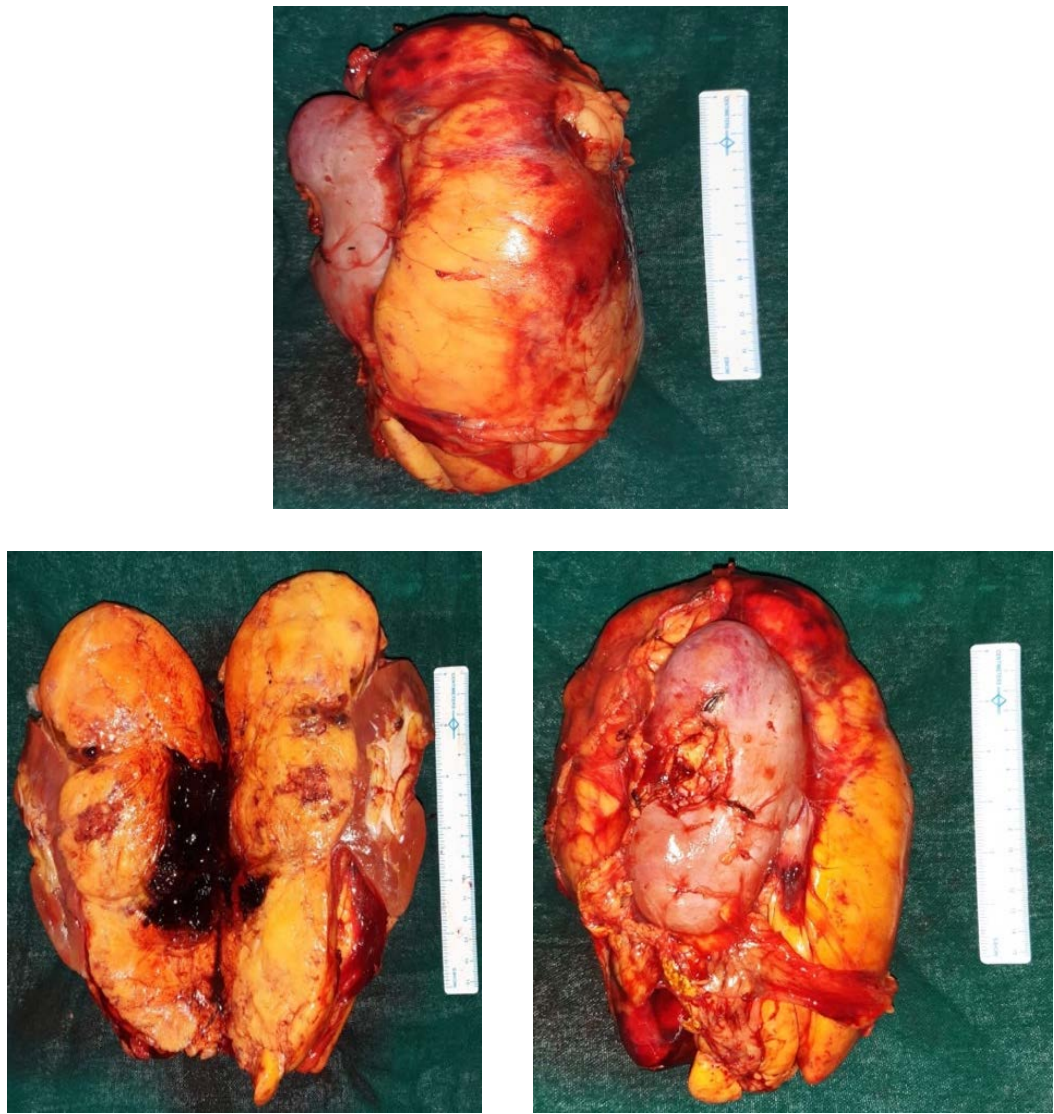

Figure 2. Left nephrectomy specimen showing giant angiomyolipoma arising from posterolateral aspect of left kidney measuring $27 \times 8.5 \times 6$ centimetres, tumour is seen indenting the lateral surface. Cut-section through the kidney and tumour revealed a large area of intratumoural hemorrhage.
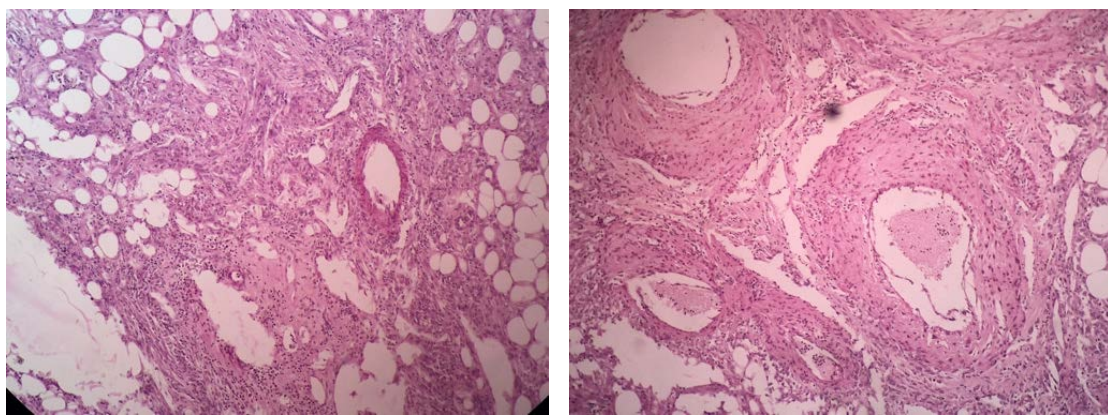

Figure 3. Histopathology sections demonstrating tumour composed of adipose tissue, thick walled blood vessels and smooth muscle. Vessel walls show prominent sclerosis.

Rare reports of angiomyolipoma mimicking renal cell carcinoma, Wilm's tumour and retroperitoneal liposarcoma are found in literature [9] [10] [11]. A few rare reports are present in which patients with ruptured angiomyolipomas presented with unexplained fever [12] [13]. In these cases, patients were known cases of bilateral angiomyolipomas with tuberous sclerosis and rupture of angiomyolipoma could be suspected. Angiomyolipoma mimicking a perinephric abscess as in our case report has not been reported in literature previously. 
The diagnosis is usually apparent and definitive based on contrast enhanced computed tomography (CECT) scan with presence of fat in the lesion (confirmed by a value of $-20 \mathrm{HU}$ or less) being a diagnostic hallmark [14]. Possibility of a fat containing renal cell cancer (RCC), fat-poor angiomyolipoma and liposarcoma need to be considered and may warrant further evaluation using MR or percutaneous biopsy in doubtful cases [15]. MRI can be used to identify the fatty tissue. However, because the presence of bleeding in any renal tumour can mimic the typical pattern of angiomyolipoma, MRI should not be considered the diagnostic method of choice [4].

With regards to the present case report, the definitive diagnosis was established following CECT. The diagnosis of angiomyolipoma was missed on USG with haemorrhage within the tumour being misdiagnosed as perinephric abscess. CECT was deferred initially to correct acute kidney injury in the patient which was possibly due to hypovolemia associated with reduced intake.

Giant AMLs, as in our case, have been deemed to be at a higher risk of rupture due to formation of intralesional aneurysms with the size of intralesional aneurysms being a strong factor predictive of haemorrhage [8].

Treatment should be individualised depending on patient factors, tumour size and symptomatology. There is no one fits all modality with regards to angiomyolipomata management.

Small and asymptomatic tumours maybe managed conservatively with active surveillance. High risk of haemorrhage associated with large angiomyolipoma warrants intervention.

Conventionally, the criteria for intervention have been symptomatic lesions, size $>4 \mathrm{~cm}$, suspicion for malignancy, women of childbearing age, an associated aneurysm size $>5 \mathrm{~mm}$, concomitant TSC and poor access to follow up or emergency care as additional considerations for treatment [8].

Modalities may include partial or radical nephrectomy and/or selective embolization. Surgery in AML has progressed from initially recommended nephrectomy to nephron sparing surgery. This is especially important in AML associated with TSC as lesions are multifocal, bilateral and recurrent. Radiofrequency ablation is another modality which has shown some promise [8].

Newer modalities have emerged to the fore with enhanced and evolving understanding of molecular basis for angiomyolipomas. Mammalian target of rapamycin (mTOR) pathway inhibitors have emerged as a novel modality. There is a use for mTOR inhibitors in tuberous sclerosis patients with giant AMLs not amenable to other treatments or patients with less remaining renal reserve; however, questions as to the durability of responses, duration of treatment and impact of toxicity from chronic therapy remain. Additionally, the role of mTOR inhibitors in the management of patients with sporadic associated giant AMLs, as in the presented case, remains to be determined [8] [16] [17].

In our case, intervention following optimisation was warranted in view of the large sized tumour and evidence of haemorrhage. Selective angioembolisation was attempted preoperatively with the intention to minimise intraoperative 
bleeding. However, embolization was unsuccessful. The patient underwent left simple nephrectomy.

Partial or nephron sparing nephrectomy and radio ablation could not be undertaken owing to the tumour encasing the whole kidney and intraoperative haemorrhage. There have been case reports of treating giant angiomyolipomas with nephron sparing surgery or excision of the tumour especially in cases of bilateral disease or solitary kidney in the settings of tuberous sclerosis [18]. Since there is further need to evaluate these approaches and our patient didn't have these special circumstances, we proceeded with the standard protocol.

\section{Conclusion}

To summarize, renal angiomyolipoma is a rare benign tumour mostly discovered as an incidental finding. The giant sized angiomyolipoma masquerading as perinephric abscess in our case report is a unique presentation, not reported previously. There is also established a need for further evaluation of other modalities of treatment in sporadic cases of giant AML with better outcomes and maximal organ preservation.

\section{Acknowledgements}

None.

\section{Conflicts of Interest}

The authors declare no conflicts of interest regarding the publication of this paper.

\section{References}

[1] Bissler, J.J. and Kingswood, J.C. (2004) Renal Angiomyolipomata. Kidney International, 66, 924-934. https://doi.org/10.1111/j.1523-1755.2004.00838.x

[2] Tsutsumi, M., Yamauchi, A., Tsukamoto, S. and Ishikawa, S. (2001) A Case of Angiomyolipoma Presenting as a Huge Retroperitoneal Mass. International Journal of Urology, 8, 470-471. https://doi.org/10.1046/j.1442-2042.2001.00344.x

[3] Obama, W., Sago, K., Owari, Y., Nozawa, T., Isurugi, K., Ohmori, S., et al. (2005) Perinephric Angiomyolipoma: A Unique Development Pattern Surrounding the Kidney. International Journal of Urology, 12, 305-307. https://doi.org/10.1111/j.1442-2042.2005.01029.x

[4] Chronopoulos, P.N., Kaisidis, G.N., Vaiopolos, C.K., Perits, D.M., Varvarousis, M.N., Malioris, A.V., et al. (2016) Spontaneous Rupture of a Giant Renal Angiomyolipoma-Wunderlich's Syndrome: Report of a Case. International Journal of Surgery Case Reports, 19, 140-143. https://doi.org/10.1016/j.ijscr.2015.12.017

[5] Seyam, R.M., Bissada, N.K., Kattan, S.A., Mokhtar, A.A., Aslam, M., Fahmy, W.E., et al. (2008) Changing Trends in Presentation, Diagnosis and Management of Renal Angiomyolipoma: Comparison of Sporadic and Tuberous Sclerosis Complex-Associated Forms. Urology, 72, 1077-1082. https://doi.org/10.1016/j.urology.2008.07.049

[6] Tamboli, P., Ro, J.Y., Amin, M.B., Ligato, S. and Ayala, A.G. (2000) Benign Tumours and Tumour-Like Lesions of the Adult Kidney. Part II: Benign Mesenchymal and 
Mixed Neoplasms, and Tumour-Like Lesions. Advances in Anatomic Pathology, 7, 47-66. https://doi.org/10.1097/00125480-200007010-00007

[7] Steiner, M.S., Goldman, S.M., Fishman, E.K. and Marshall, F.F. (1993) The Natural History of Renal Angiomyolipoma. The Journal of Urology, 150, 1782-1786.

https://doi.org/10.1016/S0022-5347(17)35895-0

[8] Flum, A.S., Hamoui, N., Said, M.A., Yang, X.J., Casalino, D.D., McGuire, B.B., Perry, K.T. and Nadler, R.B. (2016) Update on the Diagnosis and Management of Renal Angiomyolipoma. Journal of Urology, 195, 834-846. https://doi.org/10.1016/j.juro.2015.07.126

[9] Fuse, H., Ito, M., Takemura, K., Ikuta, S., Motoi, T., Okuma, T., Kataoka, M. and Koga, F. (2020) Renal Angiomyolipoma Mimicking a Well-Differentiated Retroperitoneal Liposarcoma. Case Reports in Urology, 2020, Article ID: 8812057.

https://doi.org/10.1155/2020/8812057

[10] Dhua, A.K., Ranjan, A., Agarwala, S., Bhatnagar, V., Mathur, S.R. and Devasenathipathy, K. (2017) Giant Renal Angiomyolipoma Masquerading as a Wilm's Tumour. Indian Journal of Urology, 33, 73-75.

[11] Rosenkrantz, A.B., Hecht, E.M., Taneja, S.S. and Melamed, J. (2010) Angiomyolipoma with Epithelial Cysts: Mimic of Renal Cell Carcinoma. Clinical Imaging, 34, 65-68. https://doi.org/10.1016/j.clinimag.2009.04.026

[12] Sugimoto, T., Morita, Y. and Kashiwagi, A. (2007) Spontaneous Hemorrhage from Renal Angiomyolipoma Presenting with Fever of Unknown Origin. Internal Medicine, 46, 793-794. https://doi.org/10.2169/internalmedicine.46.6361

[13] Lillo, M.E., Marín, M.D., Frutos, L., Navarro, T., Coronado, M. and Martín Curto, L.M. (2005) Angiomiolipoma renal y fiebre: Valoración mediante renograma isotópico y gammagrafía con ${ }^{67} \mathrm{Ga}$ [Renal Angiomyolipoma and Fever: Assessment with Isotopic Renogram and ${ }^{67} \mathrm{Ga}$ Scintigraphy]. Revista Española de Medicina Nuclear, 24, 322-325. https://doi.org/10.1157/13079284

[14] Jinzaki, M., Tanimoto, A., Narimatsu, Y., Kurata, T., Shinmoto, H., Hiramatsu, K., et al. (1997) Angiomyolipoma: Imaging Findings in Lesions with Minimal Fat. Radiology, 205, 497-502. https://doi.org/10.1148/radiology.205.2.9356635

[15] Lebret, T., Poulain, J.E., Moline, V., Herve, J.M., Denoux, Y., Guthrie, A., et al. (2007) Percutaneous Core Biopsy for Renal Masses: Indications, Accuracy and Results. Journal of Urology, 178, 1184-1188. https://doi.org/10.1016/j.juro.2007.05.155

[16] Davies, D.M., de Vries, P.J., Johnson, S.R., McCartney, D.L., Cox, J.A., Serra, A.L., et al. (2011) Sirolimus Therapy for Angiomyolipoma in Tuberous Sclerosis and Sporadic Lymphangioleiomyomatosis: A Phase 2 Trial. Clinical Cancer Res, 17, 4071-4081. https://doi.org/10.1158/1078-0432.CCR-11-0445

[17] Bissler, J.J., Kingswood, J.C., Radzikowska, E., Zonnenburg, B.A., Frost, M., Belousova, E., et al. (2013) Everolimus for Angiomyolipoma Associated with Tuberous Sclerosis Complex or Sporadic Lymphangioleiomyomatosis (EXIST-2): A Multicentre, Randomised, Double-Blind, Placebo-Controlled Trial. Lancet, 381, 817-824. https://doi.org/10.1016/S0140-6736(12)61767-X

[18] Shiroyanagi, Y., Kondo, T., Tomita, E., Onitsuka, S., Ryoji, O., Ito, F., Nakazawa, H. and Toma, H. (2002) Nephron-Sparing Tumorectomy for a Large Benign Renal Mass: A Case of Massive Bilateral Renal Angiomyolipomas Associated with Tuberous Sclerosis. International Journal of Urology, 9, 117-119. https://doi.org/10.1046/j.1442-2042.2002.00432.x 